\title{
Lateral and Dorsal Column Hyperintensity on Magnetic Resonance Imaging in a Patient with Myelopathy Associated with Intrathecal Chemotherapy
}

\author{
Fumine Saito ${ }^{a, c}$ Taku Hatano $^{a} \quad$ Masaaki Hori $^{c}$ Miwako Kawamura $^{a}$

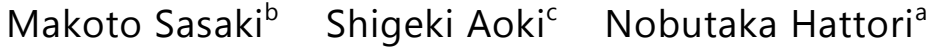 \\ ${ }^{a}$ Department of Neurology, ${ }^{b}$ Division of Hematology, Department of Internal Medicine, \\ and ${ }^{\mathrm{C}}$ Department of Radiology, Juntendo University School of Medicine, Tokyo, Japan
}

\section{Key Words}

Magnetic resonance imaging - Diffusion imaging · Spinal cord · Cytosine arabinoside ·

Methotrexate $\cdot$ Subacute combined degeneration

\begin{abstract}
Chemotherapy-related myelopathy mimicking subacute combined degeneration $(\mathrm{SCD})$ has rarely been reported. We encountered a 35-year-old female with sensory ataxia after intrathecal chemotherapy. Spinal magnetic resonance imaging showed localized abnormal signal areas in the lateral and dorsal white matter, mimicking SCD. Diffusion imaging showed restricted water diffusion and increased microstructural complexity, and cerebrospinal fluid analysis showed increased levels of myelin basic proteins, indicating demyelinating myelopathy. Advanced diffusion imaging can provide more information on the microstructure of chemotherapy-related myelopathy.
\end{abstract}

\section{Introduction}

Intrathecal chemotherapy combined with systemic chemotherapy has been used for both the prophylactic and therapeutic treatment of hematologic malignancies in the central nervous system. However, intrathecal chemotherapy has been associated with neurotoxic adverse effects, including myelopathy, encephalopathy, seizure, and cauda equina syndrome [1]. Although myelopathy is the most common adverse effect associated with intrathecal

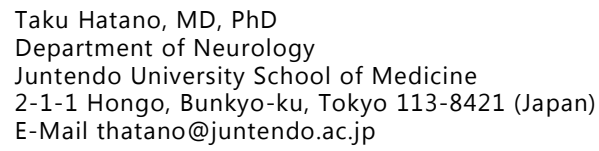


chemotherapy, magnetic resonance imaging (MRI) has rarely been reported in the literature. Here, we report a case of a 35-year-old female presenting with paraparesis, sensory ataxia, and loss of position sense in her lower limbs after systemic high-dose methotrexate and cytosine arabinoside (HD-MTX/Ara-C) therapy and intrathecal Ara-C therapy. Spinal T2weighted (T2-WI) MRI demonstrated abnormal intensity lesions in the dorsolateral column, mimicking subacute combined degeneration (SCD). In addition, we assessed diffusion tensor/kurtosis imaging (DTI/DKI) and q-space imaging (QSI) metrics in this myelopathy.

\section{Case Presentation}

A 35-year-old female underwent partial tumor resection and postoperative steroid therapy for a cerebellopontine angle tumor. Three years after treatment, she developed a headache along with vomiting. Cranial MRI revealed a lesion with abnormal intensity in the third ventricle, and transventricular endoscopic biopsy and ventriculostomy were performed. Histological examination revealed the characteristic findings of a diffuse, large B-cell lymphoma. A course of systemic HD-MTX/Ara-C treatment diminished the tumor on brain MRI and improved the clinical symptoms. The patient received an additional 3 courses of HD-MTX/Ara-C and repetitive lumbar intrathecal Ara-C (100 mg) and dexamethasone ( $4 \mathrm{mg}$ ). One month after the first round of intrathecal chemotherapy, the patient complained of bilateral numbness in her lower legs despite normal spinal MRI. She received a repetitive course of intrathecal Ara-C (40 mg) and prednisolone (4 mg) with or without intrathecal MTX (15 mg) for 6 months, along with whole-brain radiotherapy for 1 month. Nine months after the first round of intrathecal chemotherapy, the patient gradually developed an unstable gait. Neurological examination revealed a mild lower-limb weakness and a sensory ataxic gait with a positive Romberg's sign. Pinprick, light touch, vibration sensation, and proprioception were impaired below the level of the Th10 dermatome. Deep tendon reflexes were normal in all limbs, but diminished abdominal reflexes and bilateral extensor planter responses were noted. Macrocytic anemia was present: red blood cells $2.64 \times 10^{4} \mu \mathrm{l}$, hemoglobin $9.7 \mathrm{~g} / \mathrm{dl}$, mean corpuscular volume $112.5 \mathrm{fl}$, with a reduced thrombocyte count. The results of routine biochemistry, autoantibodies, and a thyroid function test were unremarkable. Her serum vitamin $B_{12}$ level was in the lower limits of normal $[286 \mathrm{pg} / \mathrm{ml}$ (normal range 180-914)], and her serum levels of folate and copper were slightly reduced [2.8 $\mathrm{ng} / \mathrm{ml}$ (normal range >3.1) and $65 \mathrm{mg} / \mathrm{dl}$ (normal range 68-128), respectively]. Cerebrospinal fluid (CSF) studies revealed a white blood cell count of $1 / \mu \mathrm{l}$, a protein level of $24 \mathrm{mg} / \mathrm{dl}$, and increased levels of myelin basic protein (MBP; $502 \mathrm{pg} / \mathrm{ml}$ ). Nerve conduction velocities and compound muscle action potentials were normal, while somatosensory evoked potentials demonstrated a normal N 20 latent time $(19.50 \mathrm{~ms})$ but prolonged P 40 responses $(44.1 \mathrm{~ms})$. The patient's spinal T2-WI MRI demonstrated abnormal signal intensity in the bilateral and dorsal columns in the thoracic cord, extending from Th3 to Th10 (fig. 1).

We performed DTI, DKI, and QSI and calculated each parameter (as described previously [2-4]), in the regions of interest on the dorsal column at Th7-9 and C3-5 of the patient, along with 4 control subjects. The results at Th7-9 of the patient and control subjects (mean \pm standard deviation) were as follows: apparent diffusion coefficient (ADC) $\left(10^{-3} \mathrm{~mm}^{2} / \mathrm{s}\right)$ : 0.55 and $1.16 \pm 0.10$; fractional anisotropy (FA): 0.41 and $0.61 \pm 0.14$; lambda $(\lambda 1)\left(10^{-3}\right.$ $\left.\mathrm{mm}^{2} / \mathrm{s}\right): 0.78$ and $2.11 \pm 0.19 ;(\lambda 2+\lambda 3) / 2\left(10^{-3} \mathrm{~mm}^{2} / \mathrm{s}\right): 0.45$ and $0.69 \pm 0.19$; full width half maximum $(\mu \mathrm{m}): 17.45$ and $20.32 \pm 0.34$; mean diffusional kurtosis (MDK): 1.65 and $0.85 \pm$ 0.15. We also obtained MDK at C3-5: 1.72, 1.21, 0.78 and $1.00 \pm 0.11$ (fig. 1, fig. 2). 
The disease course, hematological findings, and MRI suggested a diagnosis of demyelinating myelopathy due to the combined effect of intrathecal chemotherapy and vitamin and mineral deficiencies. The patient's condition did not deteriorate, but also did not improve despite therapy with intravenous administration of vitamin B12 and folic acid. Seventeen months after the first round of chemotherapy, the patient died of recurrent brain lymphoma. Permission for an autopsy was not granted.

\section{Discussion}

Myelopathy has been reported as a significant adverse effect of combination therapy with intrathecal Ara-C and systemic HD-MTX [5, 6]. Risk factors for the development of intrathecal chemotherapy-related myelopathy include a high-dose and/or frequent exposure to central nervous system-directed therapy. In most patients, paraplegia with bowel and urinary disability is commonly prominent, developing within a few days to several months after intrathecal chemotherapy. MBP levels are frequently increased in the CSF, as previously reported [7]. MRI findings of intrathecal chemotherapy-related myelopathy include high signal intensity in T2-WI within the central and posterior columns [8], cord swelling, gadolinium enhancement restricted to the lateral columns [9], and the features of arachnoiditis [10].

Our patient presented with a slowly developing weakness and an impairment of position sense in the lower limbs during repetitive intrathecal Ara-C combined with HD-MTX therapy. Her CSF cell count was unremarkable with increased MBP levels, as previously seen in myelopathy due to intrathecal chemotherapy. Conventional MRI findings consisted of abnormal signal intensity in the bilateral and dorsal columns in the thoracic cord, extending from Th3 to Th10, mimicking SCD of the spinal cord. However, our patient did not present with any other clinical features of SCD, such as peripheral neuropathy and dementia. Lu et al. [11] reported 2 cases of intrathecal MTX-related myelopathy that mimicked the MRI findings of SCD, as seen in our patient. In both patients, vitamin $B_{12}$ levels were within normal limits. In this context, myelopathy due to intrathecal chemotherapy can mimic SCD.

Breuer et al. [5] described the pathological findings of intrathecal myelopathy as microvacuolization, axonal swelling, and the loss of myelin within the white matter of the spinal cord. The mechanisms underlying chemotherapy-related myelopathy mimicking SCD are still unknown. In a rabbit model, intrathecally injected Ara-C penetrated the peripheral white matter and geniculate body in the spinal cord [12], suggesting that Ara-C may have toxic demyelinating effects on the dorsolateral columns. Additionally, our patient showed slightly low vitamin and copper levels. It is well known that although vitamin $\mathrm{B}_{12}$ and folate acid levels are in the lower limits, measuring homocysteine and methylmalonic acid levels can be useful in diagnosing patients with SCD who have not received treatment $[13,14]$. We could not confirm homocysteine and methylmalonic acid levels in our patient without vitamin treatment; therefore, the possibility that a deficiency in several vitamins and minerals may have been associated with the development of SCD should be considered in our patient [13-16]. Furthermore, MTX is a folic acid antagonist and causes chemotherapyrelated myelopathy $[1,17]$. Therefore, a deficiency in vitamins and minerals may have worsened the spinal toxicity of chemotherapy in our patient.

Diffusional MRI analysis revealed microstructural information in addition to that provided by conventional MRI. Our patient showed a decreased ADC and FA at the Th7-9 levels due to markedly decreased $\lambda 1$, which indicated the preferential restriction of water diffusion along the long tracts such as the corticospinal tract and dorsal column. Increased MDK 
indicated increased microstructural complexity. These results showed that normal and wellorganized white matter structures were damaged predominantly in the long tracts. The patient also showed an increased or decreased MDK at levels C3-5, in spite of the absence of substantial intensity abnormalities on conventional and advanced diffusion MRI. MDK may be a more sensitive parameter of microstructural changes than other measurements. Taken together, conventional and advanced MRI findings in our patient suggested that cytotoxic demyelination had occurred along the lateral and posterior columns from the lower cervical down to the middle thoracic cord.

In conclusion, we reported a case of subacute dorsolateral column myelopathy associated with intrathecal chemotherapy. Vitamin and mineral deficiencies may have played a role, in part, in the pathomechanisms of myelopathy in this patient. Spinal diffusion MRI revealed cytotoxic demyelination along the posterior columns, which assisted in the diagnosis of demyelinated myelopathy. Our findings suggest that spinal DTI, QSI, and DKI are useful tools in the diagnosis of chemotherapy-related myelopathy.

\section{Acknowledgment}

This work was supported in part by grants for Young Scientists B (to T.H.) from the Japanese Ministry of Education, Culture, Sports, Science and Technology.

\section{Disclosure Statement}

The authors declare that they have no competing interests.

\section{References}

1 Kwong YL, Yeung DY, Chan JC: Intrathecal chemotherapy for hematologic malignancies: drugs and toxicities. Ann Hematol 2009;88:193-201.

-2 Hori M, Fukunaga I, Masutani Y, Nakanishi A, Shimojo K, Kamagata K, Asahi K, Hamasaki N, Suzuki Y, Aoki S: New diffusion metrics for spondylotic myelopathy at an early clinical stage. Eur Radiol 2012;22:1797-1802.

-3 Farrell JA, Zhang J, Jones MV, Deboy CA, Hoffman PN, Landman BA, Smith SA, Reich DS, Calabresi PA, van Zijl PC: q-space and conventional diffusion imaging of axon and myelin damage in the rat spinal cord after axotomy. Magn Reson Med 2010;63:1323-1335.

4 Jensen JH, Helpern JA, Ramani A, Lu H, Kaczynski K: Diffusional kurtosis imaging: the quantification of nonGaussian water diffusion by means of magnetic resonance imaging. Magn Reson Med 2005;53:1432-1440.

5 Breuer AC, Pitman SW, Dawson DM, Schoene WC: Paraparesis following intrathecal cytosine arabinoside: a case report with neuropathologic findings. Cancer 1977;40:2817-2822.

6 Resar LM, Phillips PC, Kastan MB, Leventhal BG, Bowman PW, Civin CI: Acute neurotoxicity after intrathecal cytosine arabinoside in two adolescents with acute lymphoblastic leukemia of B-cell type. Cancer 1993;71:117-123.

-7 Watterson J, Toogood I, Nieder M, Morse M, Frierdich S, Lee Y, Moertel CL, Priest JR: Excessive spinal cord toxicity from intensive central nervous system-directed therapies. Cancer 1994;74:3034-3041.

-8 Counsel P, Khangure M: Myelopathy due to intrathecal chemotherapy: magnetic resonance imaging findings. Clin Radiol 2007;62:172-176.

9 McLean DR, Clink HM, Ernst P, Coates R, al Kawi MZ, Bohlege S, Omer S: Myelopathy after intrathecal chemotherapy. A case report with unique magnetic resonance imaging changes. Cancer 1994;73:30373040.

10 Bay A, Oner AF, Etlik O, Yilmaz C, Caksen H: Myelopathy due to intrathecal chemotherapy: report of six cases. J Pediatr Hematol Oncol 2005;27:270-272. 
Saito et al.: Lateral and Dorsal Column Hyperintensity on Magnetic Resonance

Imaging in a Patient with Myelopathy Associated with Intrathecal Chemotherapy

11 Lu CH, Yao M, Liu HM, Chen YF: MR findings of intrathecal chemotherapy-related myelopathy in two cases: mimicker of subacute combined degeneration. J Neuroimaging 2007;17:184-187.

12 Burch PA, Grossman SA, Reinhard CS: Spinal cord penetration of intrathecally administered cytarabine and methotrexate: a quantitative autoradiographic study. J Natl Cancer Inst 1988;80:1211-1216.

13 Stabler SP: Vitamin B12 deficiency. N Engl J Med 2013;368:149-160.

14 Reynolds E: Vitamin B12, folic acid, and the nervous system. Lancet Neurol 2006;5:949-960.

15 Ravakhah K, West BC: Case report: subacute combined degeneration of the spinal cord from folate deficiency. Am J Med Sci 1995;310:214-216.

16 Jaiser SR, Winston GP: Copper deficiency myelopathy. J Neurol 2010;257:869-881.

17 Vezmal S, Becker A, Bode U, Jaehde U: Biochemical and clinical aspects of methotrexate neurotoxicity. Chemotherapy 2003;49:92-104.
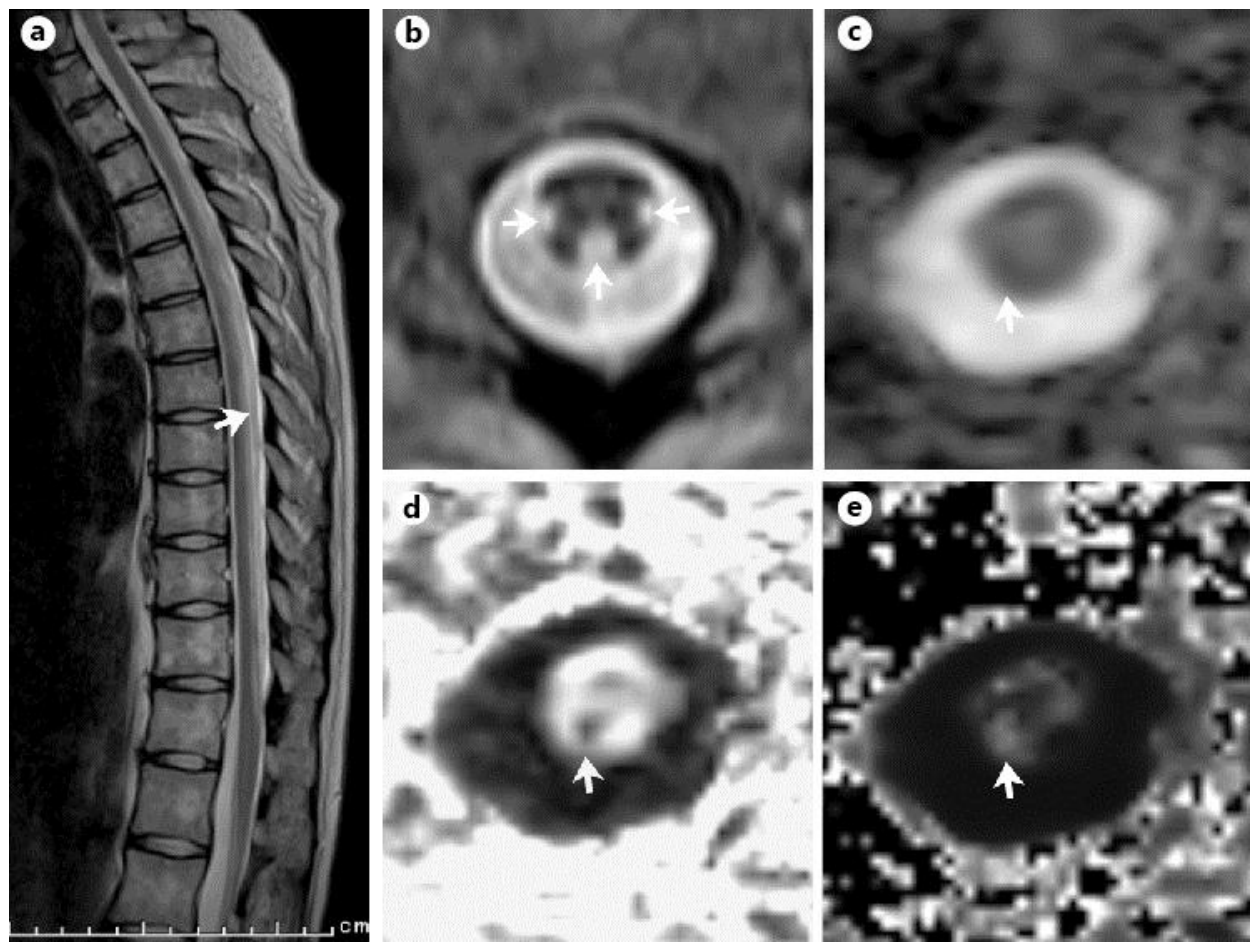

Fig. 1. Conventional and advanced diffusion MR images of the thoracic spine 9 months after the first round of intrathecal chemotherapy. a A T2-weighted sagittal image shows high signal intensity in the thoracic spine from Th3 to Th10. The arrow points to the Th8 level. b-e Axial spinal cord images at the level of Th8. b T2-weighted image showing high signal intensity in the bilateral and dorsal columns (arrows). c ADC map shows low signal intensity (decreased ADC value). d FA map shows low signal intensity (decreased FA value). e MDK map shows high signal intensity (increased MDK value) in the dorsal column (arrows in c-e). 


\section{Case Reports in Neurology}

DOI: $10.1159 / 000351848$

Saito et al.: Lateral and Dorsal Column Hyperintensity on Magnetic Resonance

Imaging in a Patient with Myelopathy Associated with Intrathecal Chemotherapy
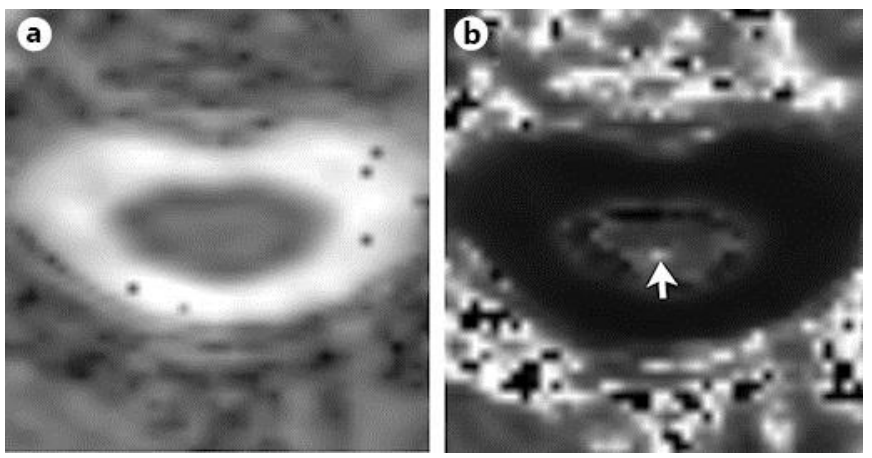

Fig. 2. Advanced diffusion axial MR images of the C3 cervical spine 9 months after the first round of intrathecal chemotherapy. a ADC values are normal. $\mathbf{b}$ Increased MDK values in the dorsal column (arrow in b). 